POS $\quad$ PROCEEDINGS

\title{
Red halos and dark baryons $\left(15^{\prime}+5^{\prime}\right)$
}

\section{Erik ZACKRISSON*}

Department of Astronomy, Stockholm University

E-mail: ezeastro.su.se

\section{Chris FLYNN}

Tuorla Observatory

E-mail: clynneutu.fi

\section{Nils BERGVALL}

Division of Astronomy \& Space Physics, Uppsala University

E-mail: nils.bergvallefysast.uu.se

\section{Genoveva MICHEVA}

Department of Astronomy, Stockholm University

E-mail: genoveva@astro.su.se

\section{Göran ÖSTLIN}

Department of Astronomy, Stockholm University

E-mail: ostlin@astro.su.se

\section{Brady CALDWELL}

Division of Astronomy \& Space Physics, Uppsala University

On cosmological scales, somewhere between one and two thirds of the baryons in the low-redshift Universe are still unaccounted for. While the baryonic fraction of the overall dark matter is subdominant on large scales, the dark baryons may have a spatial distribution that is substantially different from that of cold dark matter (CDM) and could potentially interfere with attempts to test the properties of CDM on subgalactic scales. Current models suggest that most of the dark baryons should be in the form of hot, tenuous gas, but a number of observations challenge this view, indicating that some of the missing baryons may be hiding in a cold, collapsed form. The red halos of galaxies represent one such piece that doesn't quite fit the puzzle. Over the last 15 years, deep optical/near-IR images of galaxies have revealed faint and very red structures around galaxies of different types. The colours of these halos are too extreme to be reconciled with any type of previously known stellar population, and instead indicative of a stellar population containing an abnormally high fraction of low-mass stars. Because of its high mass-to-light ratio, such a halo population could contribute substantially to the baryonic masses of galaxies, despite adding as little as a few percent to their overall light. Here, we review the current status of this field and address the constraints that direct star counts in the halo of the Milky Way impose on any putative red halo of low-mass stars around our own galaxy.

Identification of dark matter 2008

August 18-22, 2008

Stockholm, Sweden

\footnotetext{
*Speaker.
} 


\section{The Missing Baryons}

The quest to unravel the nature of dark matter, estimated to make up around $90 \%$ of the total matter content, remains one of the most important tasks of modern cosmology. Dark matter appears to exist in at least two separate forms: one baryonic, and one non-baryonic. While the non-baryonic component is the dominant one, a substantial fraction ( $1 / 3-2 / 3$, according to the latest estimates) of the baryons in the low-redshift Universe also remain at large $[1,2,3,5,6]$.

Adopting the WMAP-5 cosmological parameters [32], the cosmic density ratio of these missing baryons (hereafter synonymously referred to as baryonic dark matter or BDM) to the nonbaryonic CDM is $\Omega_{\mathrm{BDM}} / \Omega_{\mathrm{CDM}} \approx 0.09 \pm 0.03$. However, since these dark baryons may cluster differently than the CDM, the ratio of BDM to CDM densitites may become larger as one moves to smaller scales. Were this the case, it could provide an explanation for why some CDM predictions have seemingly failed on subgalactic scales. Having a sizeable baryonic contribution to the dark matter on small scales could potentially explain why many observations indicate cores in the centres of dark matter halos [7, 8, 9, 10,11] when CDM predicts cusps [12, 13, 14], and why tidal dwarf galaxies are observed to contain dark matter even though they are predicted to contain very little CDM [15].

Current models suggest that most of the missing baryons should be hiding in the warm/hot intergalactic medium [16, 17] or in the form of hot gaseous halos around galaxies [18, 19, 20], but observations are still unable to confirm this [21]. If all of the missing baryons were locked up in these components, there would be no need to worry about BDM interfering with tests designed to constrain the properties of CDM on small scales, since such extended, low-density baryon reservoirs would not lead to large BDM-to-CDM ratios in the inner regions of dark matter halos. There are, however, other possible hiding places for the missing baryons. Here, we explore the possibility that some of the missing baryons may be locked up in the form of low-mass stars in the red halos of galaxies.

\section{The Red Halos of Galaxies}

Red halos are very red and exceedingly faint structures that have been reported around galaxies of different types since the mid-1990s (see [22] for a review). The integrated colours of these structures are much too red to be reconciled with any normal type of stellar population, and instead indicative of a halo population with an abnormally high fraction of low-mass stars [23, 24, 25]. Due to their very high mass-to-light ratios, such stellar populations could contribute substantially to the baryonic masses of galaxies, even though they contribute just a few percent to their overall light [25]. Hence, red halos of low-mass could in principle provide at least a partial solution to the long-standing problem of the missing baryons.

However, the red halos remain controversial, as they are only detectable at extreme surface brightness levels, where a host of systematic problems may potentially affect the measurements $[26,27,28]$. In recent years, our team has started an ambitious project aiming to unravel the nature of these puzzling red structures. The key questions which we aim to address are:

- Are the reported halo colours correct? 
- Is the red excess due an excess of low-mass stars?

- Can this be confirmed through direct star counts?

- Are there alternative explanations for the red excess?

- How common are red halos and which types of galaxies display such structures?

- What fraction of the missing baryons do they account for?

These issues are addressed using a combination of observations, custom-designed image processing software and models. So far, this project has been awarded observing time at the $8.2 \mathrm{~m}$ ESO Very Large Telescope, the Spitzer Space Telescope, the AKARI satellite, the 3.5m ESO New Technology Telescope and the $2.5 \mathrm{~m}$ Nordic Optical Telescope.

\section{A Red Halo around the Milky Way?}

The missing baryon problem is evident within individual dark matter halos [29], and the Milky Way is no exception. The known baryonic components (thin disc, thick disk, bulge and standard stellar halo) of the Milky Way contribute around $6 \times 10^{10} M_{\odot}[30]$ to the Milky Way's virial mass of $\approx 1 \times 10^{12} M_{\odot}$ [31]. A cosmic baryon fraction of $\Omega_{\text {baryons }} / \Omega_{\mathrm{M}} \approx 0.17$ [32], combined with the theoretical prediction that the baryon fraction should be $\approx 90 \%$ of the cosmic average for a Milky Way-sized halo [33], on the other hand suggests the presence of some $\approx 1.5 \times 10^{11} M_{\odot}$ of baryonic material within its virial radius, leaving at least $60 \%$ of its baryons to be found.

Taking the red halo detections at face value, one may ask whether the Milky Way itself could be surrounded by a hitherto undetected red halo of low-mass, hydrogen-burning stars. By adopting the properties of the stacked red halo detected in the Sloan Digital Sky Survey data [34], rescaling to the dimensions of the Milky Way and assuming the bottom-heavy stellar initial mass function demonstrated to explain the colours of that structure [25], it turns out that the resulting red halo mass would be in the right ballpark (a few times $10^{10} M_{\odot}$ ) for explaining the missing baryons in the Milky Way [22].

However, if some of the missing baryons of the Milky Way are locked up in the form of hydrogen-burning stars in a red halo, such a structure must also have evaded the faint star counts aimed to constrain the luminosity function of halo subdwarfs [35, 36], since no significant excess of low-mass stars has yet been detected with this method. This is only possible if the stars of the putative red halo is locked up in star clusters [22]. While it remains a mystery how such objects would have formed, the existence of very faint (but relatively massive, probably $10^{4}-10^{5} M_{\odot}$ ) clusters of low-mass stars in halos could potentially explain some intriguing observations recently made in the halo of the nearby galaxy M60. In a search for $z \sim 6$ galaxies, Yan et al. [37] serendipitously discovered large number of faint and anomalously red point sources that seemed to cluster around M60. They argued that these objects, whatever their nature, may be responsible for the red halo phenomenon. If the point sources observed indeed are located at the distance of M60, they would have the luminosities of giants, but colours that are difficult to reconcile with current models of such stars. This could in principle be compatible with the idea of halo star clusters made primarily of low-mass stars. Making the connection is not trivial, however, since the red excess of the 
M60 objects has been detected in a photometric filter that has not yet been used in any red halo observations.

\section{Summary}

Some of the missing baryons of the Universe may in principle be hiding in the form of lowmass stars in exceedingly faint structures known as the red halos around galaxies. Existing subdwarf star counts indicate, that if a red halo of this type is to explain any non-negligible fraction of the missing baryons in the Milky Way, then the red halo stars would have to be locked up in star clusters. The existence of such star clusters could potentially explain the recent discovery of large numbers of anomalously red point sources in the halo of the nearby galaxy M60.

\section{References}

[1] Persic, M. Salucci, P. 1992, MNRAS, 258, 14

[2] Fukugita, M. 2004, Cosmic Matter Distribution: Cosmic Baryon Budget Revisited, in International Astronomical Union Symposium no. 220, ed. S. D. Ryder, D. J. Pisano, M. A. Walker, \& K. C. Freeman (San Francisco: Astronomical Society of the Pacific) p.227

[3] Fukugita, M., \& Peebles, P. J. E. 2004, ApJ, 616, 643

[4] Komatsu E., et al. 2008, $A p J S$, in press [arXiv0803.0547]

[5] Nicastro, F., et al. 2005, Nature 433, 495L

[6] Prochaska, J. X., \& Tumlinson, J. 2008, Baryons: What, When and Where?, to appear in Astrophysics in the Next Decade: JWST and Concurrent Facilities, ed. X. Tielens [arXiv:0805.4635]

[7] de Blok, W. J. G., \& Bosma, A. 2002, A\&A, 385, 816

[8] Gentile, G., Salucci, P., Klein, U., Vergani, D., Kalberla, P. 2004, MNRAS, 351, 903

[9] Zackrisson, E., Bergvall, N., Marquart, T., \& Östlin, G. 2006, A\&A, 452, 857

[10] Kuzio de Naray, R., McGaugh, S. S., de Blok, W. J. G. 2008, ApJ, 676, 920

[11] Trachternach, C., de Blok, W. J. G., Walter, F., Brinks, E., \& Kennicutt, R. C., Jr 2008, [arXiv0810.2116]

[12] Navarro, J. F., Frenk, C. S., \& White, S. D. M. 1996, ApJ, 462, 563

[13] Navarro, J. F., et al. 2004, MNRAS, 349, 1039

[14] Navarro, J. F., et al. 2008, [arXiv:0810.1522]

[15] Bournaud, F., et al. 2007, Science, 316, 1166

[16] Cen, R., \& Ostriker, J. P. 1999, ApJ, 514, 1

[17] Davé, R., et al. 2001, ApJ, 552, 473

[18] Maller, A. H., \& Bullock, J. S. 2004, MNRAS, 355, 694

[19] Fukugita, M., \& Peebles, P. J. E. 2006, ApJ, 639, 590

[20] Sommer-Larsen, J. 2006, ApJ, 644, 1 
[21] Bregman, J. N. 2007, ARA\&A, 45, 221

[22] Zackrisson, E., \& Flynn, C., 2008, ApJ, in press [arXiv0809.2991]

[23] Lequeux, J., Fort, B., Dantel-Fort, M., Cuillandre, J.-C., \& Mellier, Y. 1996, A\&A, 312, L1

[24] Rudy, R. J., Woodward, C. E., Hodge, T., Fairfield, S. W., \& Harker, D. 1997, Nature, 387, 159

[25] Zackrisson, E., Bergvall, N., Östlin, G., Micheva, G., \& Leksell, M. 2006, ApJ, 650, 812

[26] Zheng, Z., et al. 1999, AJ, 117, 2757

[27] Zackrisson, E., \& Östlin, G. 2008, Effects of extragalactic background light on deep surface photometry of galaxies, in Formation and Evolution of Galaxy Disks, ASP conference series 396, p. 227

[28] de Jong, R. S. 2008, MNRAS, 388, 1521

[29] McGaugh, S. S., The Halo by Halo Missing Baryon Problem, in Dark Galaxies and Lost Baryons, Proceedings of the International Astronomical Union, 244, p. 136

[30] Flynn, C., Holmberg, J., Portinari, L., Fuchs, B., \& Jahreiß, H. 2006, MNRAS, 372, 1149

[31] Klypin, A., Zhao, H., \& Somerville, R. S. 2002, ApJ, 573, 597

[32] Komatsu E., et al. 2008, ApJS, submitted [arXiv0803.0547]

[33] Crain, R. A., et al. 2007, MNRAS, 377, 41

[34] Zibetti, S., White, S. D. M., \& Brinkmann, J. 2004, MNRAS, 347, 556

[35] Gould, A., Flynn, C., \& Bahcall, J. N. 1998, ApJ, 503, 798

[36] Digby, A. P., Hambly, N. C., Cooke, J. A., Reid, I. N., \& Cannon, R. D. 2003, MNRAS, 344, 583

[37] Yan, H., Hathi, N. P., \& Windhorst, R. A. 2008, ApJ, 675, 136 